\title{
Avaliação do impacto da cirurgia refrativa na qualidade de vida por meio do questionário NEI-ROL (National Eye Institute Refractive Error Quality of Life)
}

\author{
Evaluation of the impact of refractive surgery on quality of life using the NEI-RQL \\ (National Eye Institute Refractive Error Quality of Life) instrument
}

\author{
Larissa Madeira Nunes ${ }^{1}$ \\ Paulo Schor ${ }^{2}$
}

Trabalho apresentado ao Departamento de Oftalmologia da Universidade Federal de São Paulo (UNIFESP), para obtenção do título de Doutor em Ciências.

${ }^{1}$ Doutora pelo programa de pós-graduação em Oftalmologia e Ciências Visuais da Universidade Federal de São Paulo (UNIFESP). São Paulo (SP).

2 Professor Livre Docente do Departamento de Oftalmologia da UNIFESP. São Paulo (SP).

Endereço para correspondência: Av. Rio Poty, 1870/ 101 - Teresina (PI) CEP 64049-410

E-mail: lmbnunes@terra.com.br

Recebido para publicação em 26.04.2005 Aprovação em 15.07.2005

Nota Editorial: Depois de concluída a análise do artigo sob sigilo editorial e com a anuência do Dr. Roberto Freda sobre a divulgação de seu nome como revisor, agradecemos sua participação neste processo.

\begin{tabular}{|l|}
\hline RESUMO \\
\hline Objetivos: Mensurar o impacto da cirurgia refrativa nos diversos aspectos \\
da qualidade de vida avaliados por meio do questionário National Eye \\
Institute Refractive Error Quality of Life(NEI-RQL). Avaliar a sensibilidade \\
da nossa versão em língua portuguesa do NEI-RQL, para detectar mu- \\
danças clinicamente significativas, decorrentes da cirurgia. Métodos: Um \\
total de 96 pacientes da Universidade Federal de São Paulo completaram \\
o NEI-RQL antes e após a realização de cirurgia fotorrefrativa, entre março \\
de 2002 e abril de 2003. Baseados nas respostas dos pacientes, foram \\
calculados escores para cada uma das 13 escalas que compõem o questio- \\
nário. Foram comparados os escores obtidos antes e após cirurgia. A \\
sensibilidade do questionário foi avaliada por meio do cálculo da magnitu- \\
de de efeito para cada escala. Resultados: Verificou-se um aumento \\
estatisticamente significante nos escores de todas as escalas do NEI-RQL. \\
O questionário mostrou-se sensível para detectar mudanças decorrentes \\
da cirurgia refrativa, tendo a magnitude de efeito variado de 0,4, na escala \\
"ofuscamento" a4,19, na escala “expectativas". Conclusões: O questioná- \\
rio NEI-RQL mostrou-se sensível para detectar mudanças clinicamente \\
significativas na qualidade de vida, decorrentes da cirurgia fotorrefrativa, \\
tendo-se verificado aumento nos escores de todas as escalas do NEI-RQL. \\
\hline
\end{tabular}

Descritores: Erros de refração/cirurgia; Cirurgia a laser; Refração ocular; Acuidade visual; Qualidade de vida; Atividades cotidianas; Satisfação do paciente; Questionários

\section{INTRODUÇÃO}

Espera-se da cirurgia oftalmológica (e da refrativa, em especial) uma melhora não apenas da acuidade visual dos pacientes, mas de diversos aspectos de suas vidas relacionados a ela, nos campos social, emocional, psicológico, físico e de bem-estar geral ${ }^{(1-4)}$. Tradicionalmente, o sucesso da cirurgia refrativa tem sido avaliado por parâmetros puramente clínicos, como acuidade visual sem correção e equivalente esférico residual. Entretanto, a importância de se incorporar a esta avaliação a perspectiva do próprio paciente em relação à sua patologia e ao tratamento médico ou cirúrgico da mesma, vem sendo amplamente reconhecida ${ }^{(4-11)}$.

Com o intuito de facilitar esta avaliação em várias áreas da oftalmologia, tem-se desenvolvido questionários que envolvem perguntas agrupadas em domínios ou escalas, abrangendo aspectos específicos da função visual e da qualidade de vida relacionada à visão ${ }^{(4-14)}$. Até o presente momento, 
existem apenas dois questionários rigorosamente submetidos a um estudo de validação, ambos americanos, especialmente desenvolvidos para a avaliação da qualidade de vida relacionada à visão em portadores de erro refrativo e, portanto, em candidatos à cirurgia refrativa: "Refractive Status and Vision Profile" (RSVP) (7) e "National Eye Institute Refractive Error Quality of Life" (NEI-RQL) ${ }^{(10,15-16)}$. Destes, o único disponível em língua portuguesa é o NEI-RQL, que foi submetido, pelos autores da presente pesquisa, a um processo de tradução, adaptação cultural e validação, tendo-se mostrado um instrumento válido e confiável para a avaliação da qualidade de vida relacionada à visão, em candidatos à cirurgia refrativa ${ }^{(17)}$.

O objetivo do presente estudo foi avaliar a sensibilidade de nossa versão em língua portuguesa do NEI-RQL para detectar alterações clinicamente significativas decorrentes da cirurgia refrativa, bem como analisar o impacto da mesma na qualidade de vida dos pacientes, por meio do questionário NEI-RQL.

\section{MÉTODOS}

Os 96 pacientes incluídos neste estudo tiveram indicação e agendamento prévios de cirurgia refrativa (LASIK ou PRK) no Setor de Cirurgia Refrativa do Departamento de Oftalmologia da Universidade Federal de São Paulo-Escola Paulista de Medicina (UNIFESP-EPM), e completaram o questionário NEI-RQL antes e após cirurgia, entre março de 2002 e abril de 2003. Todos tiveram que comprovar idade maior ou igual a 21 anos, e assinaram termo de consentimento informado, aprovado pelo Comitê de Ética da UNIFESP. Pacientes que, além do erro refracional, apresentavam doenças oculares, condições sistêmicas que pudessem alterar a estabilidade refracional (diabetes, gravidez), cirurgias oculares prévias, alterações neurológicas ou de locomoção que limitassem as atividades diárias não foram incluídos no estudo. Pacientes com melhor acuidade visual corrigida pré-operatória menor ou igual a 20/40 (0,3 LogMAR), em pelo menos um dos olhos, foram considerados amblíopes e excluídos do estudo, assim como os pacientes que não haviam completado pelo menos o nível médio de escolaridade (segundo grau).

O questionário NEI-RQL, especialmente desenvolvido para a avaliação da qualidade de vida em portadores de vícios de refração; é composto por 42 questões de múltipla escolha, agrupadas em 13 escalas ou domínios. Cada escala engloba questões relacionadas a um mesmo aspecto da qualidade de vida. As escalas do NEI-RQL são "nitidez de visão", "expectativas", "visão de perto", "visão de longe”, "flutuação de visão", "limitação de atividades", "ofuscamento", "sintomas", "dependência da correção", "correção subótima”, "aparência" e "satisfação com correção". Os pacientes foram instruídos a responder às questões do questionário, levando em consideração a correção visual que usualmente utilizavam, no caso de pacientes que necessitassem de correção. As alternativas escolhidas pelos pacientes, quando do preenchimento do questionário, eram pontuadas de 0 a 100, de acordo com as normas propostas pelos autores do questionário original ${ }^{(15)}$. O escore de cada escala era calculado pela média aritmética dos escores das questões que a compunham. Um escore global para o questionário era ainda determinado, calculando-se a média aritmética dos escores das 13 escalas. Tanto os escores das escalas como o escore global do questionário podiam variar de 0 a 100 , sendo que escores mais altos indicavam melhor qualidade de vida na escala em questão ${ }^{(15)}$.

Pacientes com cirurgia refrativa previamente indicada e agendada eram questionados pelo autor da pesquisa, durante a preparação pré-operatória no centro cirúrgico, sobre o interesse em participar do estudo. Após preenchimento de termo de consentimento informado, aprovado pelo Comitê de Ética da UNIFESP, os pacientes que iriam se submeter à cirurgia do primeiro olho eram orientados a preencher o questionário NEIRQL, baseando-se na correção visual que costumavam utilizar. Os pacientes completavam, além do questionário, uma ficha de identificação na qual constavam nome, idade, nível de escolaridade, tipo de correção utilizada, telefone, endereço e tempo de preenchimento do questionário em minutos. Os demais dados pré-operatórios, como refração sob cicloplegia e acuidade visual (AV) com e sem correção eram obtidos diretamente dos prontuários dos pacientes. Além dos retornos para pós-operatório imediato, pela rotina do serviço, os pacientes eram solicitados a retornar após 1, 3 e 6 meses, e 1 ano de pósoperatório, ocasiões em que eram realizados exames de rotina e refração sob cicloplegia. O preenchimento pós-operatório do questionário era realizado em um período mínimo de 2 e máximo de 9 meses após a cirurgia do segundo olho, nos casos em que ambos os olhos tinham indicação cirúrgica. $\mathrm{O}$ segundo questionário era aplicado sempre pelo autor da pesquisa, antes do início da consulta, para evitar que a medida da acuidade visual pudesse influenciar as respostas. As respostas pré e pós-operatórias eram convertidas em escores para cada questão, e uma média dos escores de cada escala era calculada, somando-se os escores das questões dentro desta, e dividindo-se pelo número de questões que a compunham. Desta forma eram obtidos escores pré e pós-operatórios para cada escala do NEI-RQL. No caso de pacientes que apresentavam AV sem correção (AV s/c) pré-operatória no olho contralateral de 20/30, ou melhor, em que havia sido indicada cirurgia unilateral, o segundo questionário era aplicado após pelo menos dois meses da cirurgia, assim como nos casos de reoperação. Nos poucos casos de pacientes residentes fora do Estado, ou que estivessem impossibilitados de comparecer à consulta, era enviado pelo correio, após contato telefônico, questionário pós-operatório em envelope previamente selado para resposta. Nestes casos, se alguma consulta pós-operatória constasse no prontuário, os dados pós-operatórios (AV com e sem correção e refração) eram obtidos da data mais próxima ao preenchimento do questionário.

Análise estatística descritiva foi utilizada na caracterização demográfica dos pacientes e dos dados pré e pós-operatórios relacionados a erro refrativo (média do equivalente esférico), média dos escores e tempo médio de aplicação dos 
questionários. Na comparação dos escores pré e pós-operatórios, foram calculadas a variação absoluta (diferença entre a média pós e a média pré-operatória) e a variação porcentual para cada escala. Foi determinado ainda o porcentual de pacientes que apresentaram aumento, diminuição, ou que mantiveram os escores inalterados após cirurgia, para cada escala. A sensibilidade do questionário foi avaliada por meio do cálculo da magnitude de efeito para cada escala.

\section{RESULTADOS}

Dos 102 pacientes arrolados inicialmente neste estudo, 6 foram excluídos (3 devido à perda de seguimento e 3 foram considerados amblíopes), e as análises foram realizadas para um total de 96 pacientes. Destes, 54,2\% eram do sexo feminino, $83,3 \%$ apresentavam idade menor que 40 anos, 53,1\% tinham nível superior completo ou incompleto e 59,4\% usavam apenas óculos como correção pré-operatória (Tabela 1). A grande maioria dos pacientes era míope $(93,75 \%)$, e apenas $6(6,25 \%)$ eram hipermétropes. O tempo médio gasto na aplicação do questionário foi de 15,2 minutos antes e 14,0 minutos após cirurgia.

Comparando-se os escores pré e pós-operatórios dos questionários, observa-se que em todas as escalas do NEIRQL, assim como na escala global, os valores pós-operatórios foram significativamente maiores ( $\mathrm{p}<0,01)$ (Tabela 2).

As maiores diferenças entre as médias dos escores pré e pósoperatórios foram verificadas, em ordem decrescente, nas escalas "expectativas", "dependência da correção", "aparência", "limitação de atividades", "satisfação com correção", "preocupação" e escala global. Nas escalas "ofuscamento", "nitidez de visão" e "sintomas" foram observadas as menores diferenças entre as médias dos escores pré e pós-operatórios (Tabela 2).

A tabela 3 apresenta o porcentual de pacientes que obtiveram melhora (aumento no escore), piora (diminuição no escore), ou mantiveram-se inalterados, em cada uma das escalas do questionário. As maiores porcentagens de pacientes apre-

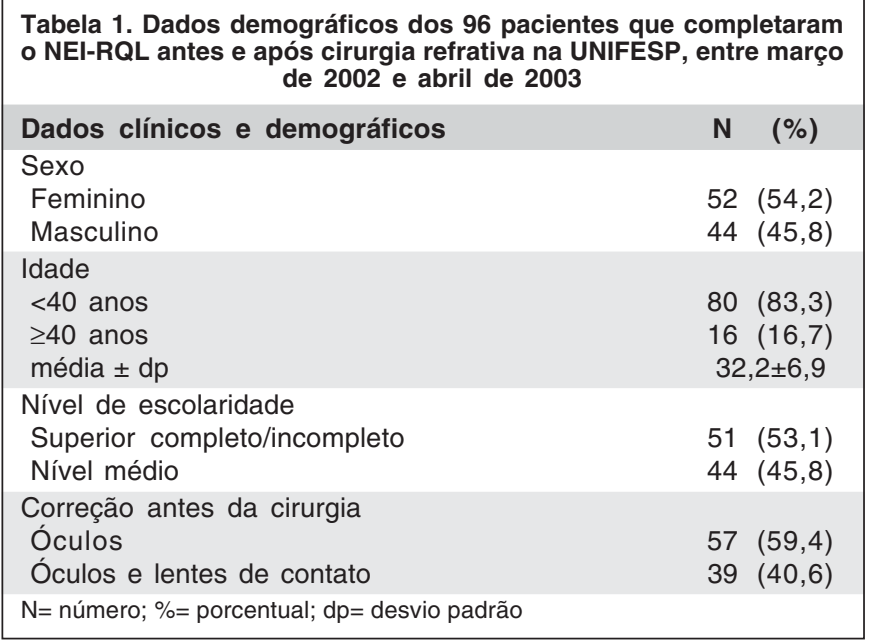

sentando melhora foram observadas nas escalas: global (100\%), "expectativas" e "dependência da correção" (99\%), "aparência" (96,9\%), "limitação de atividades" (92,7\%) e "satisfação com correção" (85,3\%). As maiores porcentagens de piora ocorreram nas escalas "sintomas" (27,1\%), "nitidez de visão" (26\%) e "ofuscamento" (25\%).

A sensibilidade do questionário em detectar alterações clinicamente significativas decorrentes da cirurgia refrativa ("responsiveness") foi avaliada pelo cálculo da magnitude de efeito

\begin{tabular}{|c|c|c|c|}
\hline \multirow[t]{3}{*}{ Escalas } & \multicolumn{2}{|c|}{$\begin{array}{l}\text { Escores } \\
\end{array}$} & \multirow{3}{*}{$\begin{array}{l}\text { Teste de } \\
\text { Wilcoxon }\end{array}$} \\
\hline & Pré-operatório & Pós-operatório & \\
\hline & $\begin{array}{c}\text { Média (dp) } \\
\text { Mediana } \\
\text { Mínimo/Máximo }\end{array}$ & $\begin{array}{c}\text { Média (dp) } \\
\text { Mediana } \\
\text { Mínimo/Máximo }\end{array}$ & \\
\hline $\begin{array}{l}\text { Nitidez de } \\
\text { visão }\end{array}$ & $\begin{array}{c}79,2(19,0) \\
84,4 \\
20,8 / 100,0\end{array}$ & $\begin{array}{c}90,5(11,8) \\
91,7 \\
47,9 / 100,0\end{array}$ & $\mathrm{p}<0,001^{*}$ \\
\hline Expectativas & $\begin{array}{c}9,9(19,4) \\
0,0 \\
0,0 / 75,0\end{array}$ & $\begin{array}{c}91,1(20,5) \\
100,0 \\
0,0 / 100,0\end{array}$ & $p<0,001^{*}$ \\
\hline $\begin{array}{l}\text { Visão de } \\
\text { perto }\end{array}$ & $\begin{array}{c}75,5(20,6) \\
78,1 \\
27,1 / 100,0\end{array}$ & $\begin{array}{c}93,9(11,8) \\
100,0 \\
33,3 / 100,0\end{array}$ & $p<0,001^{*}$ \\
\hline $\begin{array}{l}\text { Visão de } \\
\text { longe }\end{array}$ & $\begin{array}{c}72,7(21,6) \\
76,7 \\
16,7 / 100,0\end{array}$ & $\begin{array}{c}93,0(7,8) \\
93,3 \\
63,3 / 100,0\end{array}$ & $\mathrm{p}<0,001^{*}$ \\
\hline $\begin{array}{l}\text { Flutuação de } \\
\text { visão }\end{array}$ & $\begin{array}{c}62,6(28,8) \\
66,7 \\
0,0 / 100,0\end{array}$ & $\begin{array}{c}85,2(17,4) \\
87,5 \\
29,2 / 100,0\end{array}$ & $\mathrm{p}<0,001^{*}$ \\
\hline $\begin{array}{l}\text { Limitação de } \\
\text { atividades }\end{array}$ & $\begin{array}{c}53,3(28,7) \\
50,0 \\
0,0 / 100,0\end{array}$ & $\begin{array}{c}100,0(0,0) \\
100,0 \\
100,0 / 100,0\end{array}$ & $\mathrm{p}<0,001^{*}$ \\
\hline Ofuscamento & $\begin{array}{c}67,7(30,4) \\
75,0 \\
0,0 / 100,0\end{array}$ & $\begin{array}{c}79,8(24,5) \\
87,5 \\
0,0 / 100,0\end{array}$ & $\mathrm{p}<0,001^{*}$ \\
\hline Sintomas & $\begin{array}{c}74,9(18,7) \\
76,8 \\
25,0 / 100,0\end{array}$ & $\begin{array}{c}84,2(14,1) \\
84,7 \\
35,7 / 100,0\end{array}$ & $\mathrm{p}<0,001^{*}$ \\
\hline $\begin{array}{l}\text { Dependência } \\
\text { da correção }\end{array}$ & $\begin{array}{c}34,7(24,4) \\
16,7 \\
0,0 / 100,0\end{array}$ & $\begin{array}{c}96,4(11,6) \\
100,0 \\
50,0 / 100,0\end{array}$ & $\mathrm{p}<0,001^{*}$ \\
\hline Preocupação & $\begin{array}{c}22,1(27,4) \\
12,5 \\
0,0 / 100,0\end{array}$ & $\begin{array}{c}57,9(35,5) \\
62,5 \\
0,0 / 100,0\end{array}$ & $\mathrm{p}<0,001^{*}$ \\
\hline $\begin{array}{l}\text { Correção } \\
\text { subótima }\end{array}$ & $\begin{array}{c}75,3(31,5) \\
87,5 \\
0,0 / 100,0\end{array}$ & $\begin{array}{c}100,0(0,0) \\
100,0 \\
100,0 / 100,0\end{array}$ & $\mathrm{p}<0,001^{*}$ \\
\hline Aparência & $\begin{array}{c}40,9(27,8) \\
40,0 \\
0,0 / 100,0\end{array}$ & $\begin{array}{c}99,4(1,9) \\
100,0 \\
93,3 / 100,0\end{array}$ & $\mathrm{p}<0,001^{*}$ \\
\hline $\begin{array}{l}\text { Satisfação } \\
\text { com correção }\end{array}$ & $\begin{array}{c}54,6(28,2) \\
60,0 \\
0,0 / 100,0\end{array}$ & $\begin{array}{c}91,9(11,1) \\
100,0 \\
60,0 / 100,0\end{array}$ & $\mathrm{p}<0,001^{*}$ \\
\hline Global & $\begin{array}{c}55,6(13,2) \\
54,5 \\
17,2 / 81,5\end{array}$ & $\begin{array}{c}89,5(7,8) \\
90,2 \\
66,6 / 100,0\end{array}$ & $\mathrm{p}<0,001^{*}$ \\
\hline
\end{tabular}


("effect size") para as diversas escalas. As medidas de magnitude de efeito variaram de 0,40 , na escala "ofuscamento", a 4,19, na escala "expectativas". De acordo com a classificação estabelecida por Coehn ${ }^{(18)}$ a ME foi considerada pequena (ME <0,5) para a escala "ofuscamento", mediana (ME de 0,5 a 0,7) para "nitidez de visão", "flutuação de visão", "sintomas" e "correção subótima", e grande ( $\mathrm{ME} \geq 0,8)$ para as escalas "expectativas", global, "dependência da correção", "aparência", "limitação de atividades", "preocupação", "satisfação com correção", "visão de longe" e visão de perto" (Tabela 4).

Ao se avaliar a correlação entre parâmetros pré-operatórios, verificou-se correlação positiva significante entre os escores global e da escala "satisfação com correção"; entretanto, não houve correlação entre as referidas escalas e o equivalente esférico pré-operatório do melhor e do pior olho (Tabela 5).

Analisando os mesmos parâmetros em relação ao pósoperatório, também se observou correlação positiva estatisticamente significante entre os escores da escala "satisfação com correção" e o escore global. Ao se correlacionar estes escores com medidas clínicas como EE e acuidade visual sem

\begin{tabular}{|c|c|c|c|}
\hline \multirow[t]{2}{*}{ Escalas } & \multicolumn{3}{|c|}{ Pós - Pré $(\Delta)$} \\
\hline & $\begin{array}{c}\text { Melhora } \\
\text { N } \\
(\%)\end{array}$ & $\begin{array}{c}\text { Inalterado } \\
\mathrm{N} \\
(\%)\end{array}$ & $\begin{array}{c}\text { Piora } \\
N \\
(\%)\end{array}$ \\
\hline $\begin{array}{l}\text { Nitidez de } \\
\text { visão }\end{array}$ & $\begin{array}{c}56 \\
(58,3)\end{array}$ & $\begin{array}{c}15 \\
(15,6)\end{array}$ & $\begin{array}{c}25 \\
(26,0)\end{array}$ \\
\hline Expectativas & $\begin{array}{c}95 \\
(99,0)\end{array}$ & $\begin{array}{c}0 \\
(0,0)\end{array}$ & $\begin{array}{c}1 \\
(1,0)\end{array}$ \\
\hline Visão de perto & $\begin{array}{c}66 \\
(68,8)\end{array}$ & $\begin{array}{c}17 \\
(17,7)\end{array}$ & $\begin{array}{c}13 \\
(13,5)\end{array}$ \\
\hline Visão de longe & $\begin{array}{c}73 \\
(76,0)\end{array}$ & $\begin{array}{c}13 \\
(13,5)\end{array}$ & $\begin{array}{c}10 \\
(10,4)\end{array}$ \\
\hline $\begin{array}{l}\text { Flutuação de } \\
\text { visão }\end{array}$ & $\begin{array}{c}62 \\
(64,6)\end{array}$ & $\begin{array}{c}17 \\
(17,7)\end{array}$ & $\begin{array}{c}17 \\
(17,7)\end{array}$ \\
\hline $\begin{array}{l}\text { Limitação de } \\
\text { atividades }\end{array}$ & $\begin{array}{c}89 \\
(92,7)\end{array}$ & $\begin{array}{c}7 \\
(7,3)\end{array}$ & $\begin{array}{c}0 \\
(0,0)\end{array}$ \\
\hline Ofuscamento & $\begin{array}{c}46 \\
(47,9)\end{array}$ & $\begin{array}{c}26 \\
(27,1)\end{array}$ & $\begin{array}{c}24 \\
(25,0)\end{array}$ \\
\hline Sintomas & $\begin{array}{c}63 \\
(65,6)\end{array}$ & $\begin{array}{c}8 \\
(8,3)\end{array}$ & $\begin{array}{c}26 \\
(27,1)\end{array}$ \\
\hline $\begin{array}{l}\text { Dependência } \\
\text { da correção }\end{array}$ & $\begin{array}{c}95 \\
(99,0)\end{array}$ & $\begin{array}{c}1 \\
(1,0)\end{array}$ & $\begin{array}{c}0 \\
(0,0)\end{array}$ \\
\hline Preocupação & $\begin{array}{c}65 \\
(67,7)\end{array}$ & $\begin{array}{c}23 \\
(24,0)\end{array}$ & $\begin{array}{c}8 \\
(8,3)\end{array}$ \\
\hline $\begin{array}{l}\text { Correção } \\
\text { subótima }\end{array}$ & $\begin{array}{c}49 \\
(51,0)\end{array}$ & $\begin{array}{c}47 \\
(49,0)\end{array}$ & $\begin{array}{c}0 \\
(0,0)\end{array}$ \\
\hline Aparência & $\begin{array}{c}93 \\
(96,9)\end{array}$ & $\begin{array}{c}3 \\
(3,1)\end{array}$ & $\begin{array}{c}0 \\
(0,0)\end{array}$ \\
\hline $\begin{array}{l}\text { Satisfação } \\
\text { com correção }\end{array}$ & $\begin{array}{c}79 \\
(85,3)\end{array}$ & $\begin{array}{c}14 \\
(14,6)\end{array}$ & $\begin{array}{c}3 \\
(3,1)\end{array}$ \\
\hline Global & $\begin{array}{c}96 \\
(100,0)\end{array}$ & $\begin{array}{c}0 \\
(0,0)\end{array}$ & $\begin{array}{c}0 \\
(0,0)\end{array}$ \\
\hline
\end{tabular}

correção (AV s/c) no melhor e no pior olho, verificou-se apenas correlação negativa fraca, estatisticamente significante, entre os escores citados e a AV s/c no pior olho. As demais correlações mostraram-se não significantes (Tabela 6).

\section{DISCUSSÃO}

Ainda são raros, na literatura nacional, e em especial no campo da Oftalmologia, estudos envolvendo questionários e qualidade de vida. Em nenhum deles, os portadores de vícios de refração ou candidatos à cirurgia refrativa foram a população alvo do estudo ${ }^{(19-21)}$. Felizmente, a importância de se incorporar à avaliação de parâmetros puramente clínicos rotineiramente empregados, a perspectiva do próprio paciente em relação à sua patologia e ao tratamento médico ou cirúrgico da mesma, vem sendo amplamente reconhecida ${ }^{(4-11)}$. Dos instrumentos desenvolvidos para a avaliação da qualidade de vida relacionada à visão em portadores de erro refrativo, o único disponível em versão traduzida e culturalmente adaptada para uso em língua portuguesa é o National Eye Institute Refractive Error Quality of Life (NEI-RQL), que foi submetido a um processo formal de validação em estudo transversal ${ }^{(17)}$. Como um dos principais objetivos dos questionários que avaliam qualidade de vida em candidatos à cirurgia refrativa é detectar mudanças significativas decorrentes da mesma, prosseguimos com a avaliação do questionário, em estudo longitudinal, comparando resultados obtidos antes e após cirurgia.

Verificamos em todas as escalas do NEI-RQL, assim como na escala global, um aumento estatisticamente significante nos escores após cirurgia (Tabela 2), indicando melhora na qualidade de vida, nos diversos aspectos avaliados pelo questionário. O escore global médio aumentou de 55,6 para 89,5 , e a média do escore da escala "satisfação com correção" passou de 54,6 para 91,9, após cirurgia. As escalas em que se observou maior ganho nos escores (variação > 25) foram: "expectativas", "dependência da correção", "aparência", "limitação de atividades", "satisfação", "preocupação", e escala global (Tabela 4). O menor ganho em escores foi observado nas escalas "ofuscamento", "nitidez de visão" e "sintomas". Estes resultados são muito semelhantes aos obtidos com a aplicação do questionário original, americano, em estudo multicêntrico, no qual se verificou aumento estatisticamente significante em todas as escalas, com exceção da "nitidez de visão", que apresentou ganho não estatisticamente significante, e "ofuscamento", escala em que foi observada diminuição significante nos escores após cirurgia ${ }^{(11)}$. Ainda no estudo citado, as escalas cujos escores apresentaram maior aumento após cirurgia foram as mesmas da presente pesquisa, com exceção da escala "preocupação".

Motivados pela noção de que medidas como média e mediana nem sempre refletem, de maneira fidedigna, o que ocorre com cada paciente individualmente, calculamos o porcentual de pacientes que obtiveram melhora (aumento no escore), piora (diminuição no escore), ou mantiveram-se inalterados, 


\begin{tabular}{|c|c|c|c|c|}
\hline \multirow[t]{3}{*}{ Escalas } & \multicolumn{3}{|c|}{ Avaliação } & \multirow{3}{*}{$\begin{array}{l}\text { Magnitude } \\
\text { de efeito }\end{array}$} \\
\hline & Pré-operatória & Pós-operatória & Variação absoluta & \\
\hline & Média (dp) - mediana & Média (dp) - mediana & Média (dp) - mediana & \\
\hline Expectativas & $9,9(19,4)-0,0$ & $91,1(20,5)-100,0$ & $81,3(27,9)-100,0$ & 4,19 \\
\hline Visão de perto & $75,5(20,6)-78,1$ & $93,9(11,8)-100,0$ & $18,4(22,0)-14,6$ & 0,89 \\
\hline Visão de longe & $72,7(21,6)-76,7$ & $93,0(7,8)-93,3$ & $20,3(22,0)-15,8$ & 0,94 \\
\hline Limitação de atividades & $53,3(28,7)-50,0$ & $100,0(0,0)-100,0$ & $46,7(28,7)-50,0$ & 1,63 \\
\hline Ofuscamento & $67,7(30,4)-75,0$ & $79,8(24,5)-87,5$ & $12,1(33,5)-0,0$ & 0,40 \\
\hline Sintomas & $74,9(18,7)-76,8$ & $84,2(14,1)-85,7$ & $9,3(19,4)-7,1$ & 0,50 \\
\hline Dependência da correção & $34,7(24,4)-16,7$ & $96,4(11,6)-100,0$ & $61,7(26,6)-70,8$ & 2,53 \\
\hline Preocupação & $22,1(27,4)-12,5$ & $57,9(35,5)-62,5$ & $35,8(37,6)-25,0$ & 1,31 \\
\hline Correção subótima & $75,3(31,5)-87,5$ & $100,0(0,0)-100,0$ & $24,7(31,5)-12,5$ & 0,78 \\
\hline
\end{tabular}

\begin{tabular}{|c|c|c|c|}
\hline $\begin{array}{l}\text { Parâmetros } \\
\text { pré-operatórios }\end{array}$ & $\begin{array}{l}\text { Escore } \\
\text { global }\end{array}$ & $\begin{array}{l}\text { Módulo EE no } \\
\text { melhor olho }\end{array}$ & $\begin{array}{l}\text { Módulo EE } \\
\text { no pior olho }\end{array}$ \\
\hline $\begin{array}{l}\text { Satisfação com } \\
\text { correção }\end{array}$ & $\begin{array}{l}r_{s}=0,45 \\
p<0,001^{*}\end{array}$ & $\begin{array}{l}r_{s}=0,12 \\
p=0,262\end{array}$ & $\begin{array}{l}r_{s}=0,06 \\
p=0,575\end{array}$ \\
\hline Escore global & & $\begin{array}{l}r_{s}=0,16 \\
p=0,126\end{array}$ & $\begin{array}{l}r_{s}=0,14 \\
p=0,165\end{array}$ \\
\hline
\end{tabular}

em cada uma das escalas do questionário (Tabela 3). As maiores porcentagens de pacientes apresentando melhora foram observadas nas escalas: global (100\%), "expectativa" e "dependência da correção" (99\%), "aparência” (96,9\%), "limitação de atividades" $(92,7 \%)$ e "satisfação com correção" $(85,3 \%)$. As maiores porcentagens de piora ocorreram nas escalas "sintomas" (27,1\%), "nitidez de visão" (26\%) e "ofuscamento" (25\%), mostrando que, embora tenha havido melhora estatisticamente significante em todas as escalas, existem pequenos "subgrupos" de pacientes que apresentaram piora em escalas específicas como as supracitadas. As porcentagens de piora nas escalas "nitidez de visão" (26\%) e "ofuscamento" (25\%) sugerem que o efeito de eventuais ametropias residuais e/ou de aberrações de alta ordem provocadas pela cirurgia, ainda constituem importante terreno para pesquisas e avanços futuros em cirurgia refrativa. Instrumentos como o NEI-RQL oferecem elementos essenciais para ampliar o nosso conhecimento sobre os resultados subjetivos da cirurgia refrativa, especialmente em relação às possíveis fontes de insatisfação dos pacientes ${ }^{(11)}$.

Embora porcentagens consideráveis de pacientes tenham apresentado piora em algumas escalas ("sintomas", "nitidez de visão", "ofuscamento"), apenas 3,1\% apresentaram piora na escala "satisfação com correção", o que mostra que a cirurgia refrativa teve grande impacto positivo na satisfação dos pacientes. Diversos estudos a respeito dos resultados subjetivos da cirurgia fotorrefrativa também relataram altos índices de satisfação. Entretanto, alguns são limitados a meros relatos de satisfação e sintomas, utilizando questionários não validados, com um pequeno número de questões que não abrangem os diversos aspectos da qualidade de vida relacionados à visão ${ }^{(2-3,6,22-26)}$. Tais estudos podem omitir informações importantes sobre aspectos específicos e eventuais efeitos colaterais negativos da cirurgia.

Vale ressaltar que a satisfação dos pacientes tende a ser tanto maior, quanto mais realistas forem as expectativas dos pacientes, onde o oftalmologista assume importante papel de orientação sobre o objetivo primordial da cirurgia de tornar o paciente socialmente independente do uso de correção visual, e não necessariamente de transformá-lo em um indivíduo emétrope ${ }^{(27)}$.

Estudos mais completos, com questionários mais extensos, avaliando diferentes aspectos da satisfação pós-operatória e da qualidade de vida relacionada à visão tem sido relata$\operatorname{dos}^{(4,28-30)}$, muitos, entretanto, consistem em estudos retrospectivos $^{(28-30)}$ nos quais os questionários foram aplicados apenas no período pós-operatório, o que constitui importante fator limitante na avaliação das mudanças decorrentes da cirurgia, visto que não se dispõe de um padrão pré-operatório para comparações. Poucos estudos prospectivos utilizando questionários foram realizados para avaliar mudanças nos diferentes aspectos da qualidade de vida relacionada à visão após cirurgia refrativa ${ }^{(4,9,11,31)}$ destes, apenas dois utilizaram questionários com propriedades psicométricas (confiabilidade e validade) devidamente comprovadas ${ }^{(7,16)}$.

O presente estudo é o primeiro realizado no Brasil, com questionário culturalmente adaptado e validado para a língua 


\begin{tabular}{|c|c|c|c|c|c|}
\hline $\begin{array}{l}\text { Parâmetros } \\
\text { pós-operatórios }\end{array}$ & $\begin{array}{l}\text { Escore global } \\
\qquad(n=96)\end{array}$ & $\begin{array}{l}\text { Módulo(EE) } \\
\text { melhor olho } \\
(n=93)\end{array}$ & $\begin{array}{l}\text { Módulo(EE) } \\
\text { pior olho } \\
(n=93)\end{array}$ & $\begin{array}{c}\text { AV s/c (logMAR) } \\
\text { melhor olho } \\
(n=94)\end{array}$ & $\begin{array}{c}\text { AV s/c (logMAR) } \\
\text { pior olho } \\
(n=94)\end{array}$ \\
\hline Satisfação com correção & $\begin{array}{l}r_{s}=0,67 \\
p<0,001^{*}\end{array}$ & $\begin{array}{l}r_{s}=-0,07 \\
p=0,515\end{array}$ & $\begin{array}{l}r_{s}=-0,03 \\
p=0,764\end{array}$ & $\begin{array}{l}r_{s}=-0,18 \\
p=0,087\end{array}$ & $\begin{array}{l}r_{s}=-0,29 \\
p=0,005^{\star}\end{array}$ \\
\hline Escore global & - & $\begin{array}{l}r_{s}=0,01 \\
p=0,910\end{array}$ & $\begin{array}{l}r_{s}=0,06 \\
p=0,543\end{array}$ & $\begin{array}{l}r_{s}=-0,18 \\
p=0,089\end{array}$ & $\begin{array}{l}r_{s}=-0,25 \\
p=0,017^{*}\end{array}$ \\
\hline
\end{tabular}

portuguesa, a avaliar, prospectivamente, os resultados subjetivos da cirurgia fotorrefrativa, no que se refere aos diversos aspectos da qualidade de vida relacionados à visão.

Mais do que simplesmente descrever os resultados subjetivos da cirurgia, o principal objetivo da presente pesquisa era determinar se o NEI-RQL, questionário por nós utilizado, era suficientemente sensível para detectar mudanças clinicamente significativas decorrentes da cirurgia refrativa ("responsiveness"). Esta avaliação foi realizada por meio do cálculo da magnitude de efeito (ME) para cada uma das escalas do questionário. A ME é a medida padrão da sensibilidade de um questionário e fornece uma estimativa da magnitude de mudança decorrente de uma intervenção. Constitui um instrumento para complementar os testes de significância estatística, e facilitar a interpretação da magnitude das mudanças clinicamente significativas no estado de saúde, permitindo ainda comparações entre diferentes estudos, quando questionários semelhantes forem utilizados. Pode ser calculada dividindo-se a variação média verificada nos escores pelo desvio padrão dos mesmos antes da intervenção ${ }^{(32)}$.

Neste estudo, as medidas de ME variaram de 0,40, na escala "ofuscamento", a 4,19, na escala "expectativas". De acordo com a classificação estabelecida por Cohen ${ }^{(18)}$, a magnitude de efeito ("effect size") foi considerada pequena $(<0,5)$ para a escala "ofuscamento"; mediana $(0,5$ a 0,7$)$ para "nitidez de visão", "flutuação de visão", "sintomas" e "correção subótima"; e grande $(\geq 0,8)$ para as escalas "expectativas", escala global, "dependência da correção", "aparência", "limitação de atividades", "preocupação", "satisfação com correção", "visão de longe" e "visão de perto" (Tabela 4).

No estudo americano em que o NEI-RQL original foi aplicado a 185 pacientes, antes e após cirurgia refrativa, as escalas do questionário também se mostraram bastante sensíveis para detectar alterações decorrentes da cirurgia, com a maioria das escalas apresentando uma magnitude de efeito mediana ("visão de longe", "preocupação", "correção subótima”) ou grande ("expectativas", "limitação de atividades", "dependência da correção", "aparência" e "satisfação com correção"). As escalas "nitidez de visão", "visão de perto", "flutuação de visão", "ofuscamento" e "sintomas" apresentaram pequena magnitude de efeito ${ }^{(11)}$.

Relatos demonstram que as escalas do questionário RSVP também se mostraram sensíveis, em graus variados, às mudan- ças em resposta à cirurgia refrativa; a escala global teve grande magnitude de efeito, enquanto a escala relacionada à habilidade de dirigir teve a menor magnitude de efeito. Os autores ressaltam, entretanto, que o fato de uma escala em particular apresentar pequena ME não necessariamente implica na irrelevância das informações obtidas por meio dela. No cálculo da ME, o numerador consiste na variação média dos escores após cirurgia. Portanto, se iguais porcentagens de pacientes apresentarem melhora e piora nos escores em determinada escala, o numerador e, consequentemente, a ME da referida escala se aproximam de zero. Embora escalas relacionadas a ofuscamento e problemas para dirigir tenham apresentado menor magnitude de efeito, os autores consideraram que não seria coerente retirar tais tópicos da avaliação dos resultados subjetivos da cirurgia refrativa ${ }^{(9,33)}$.

Schein alerta ainda para o fato de que o uso isolado do escore global de um questionário que avalia múltiplos aspectos da qualidade de vida deve ser analisado sempre levando em consideração as diversas escalas, pois o escore global pode mostrar grande aumento, mesmo que tenha ocorrido piora significativa (isto é, importante para o paciente) em uma ou mais das escalas, individualmente ${ }^{(33)}$. De fato, uma das críticas que se poderia fazer à escala global do NEI-RQL, por exemplo, consiste na forma como seu escore é calculado, através da média aritmética dos escores das 13 escalas, que têm, todas, portanto, o mesmo peso no escore global. Na realidade, não se deve esperar que cada aspecto da qualidade de vida relacionada à visão tenha a mesma importância para todos os pacientes. A escala "ofuscamento" provavelmente terá relevância maior para um paciente cuja profissão seja motorista profissional do que para uma dona de casa, assim como a escala "aparência" pode ter maior ou menor importância de acordo com aspectos psicológicos de cada paciente. Seria interessante dispor de uma escala global calculada a partir de uma média ponderada, onde cada paciente atribuísse pesos específicos às escalas, de acordo com suas necessidades, atividades, limitações e interesses. Em estudo sobre questionários que quantificam a qualidade de vida relacionada à saúde, Gill e Feinstein sugerem que a importância relativa atribuída aos diferentes aspectos avaliados deve ser sempre proveniente dos próprios pacientes envolvidos no estudo ${ }^{(34)}$.

Ao avaliar a correlação entre parâmetros pré-operatórios, 
verificou-se correlação positiva significante entre os escores global e da escala "satisfação com correção"; entretanto, não houve correlação entre as referidas escalas e o equivalente esférico pré-operatório do melhor e do pior olho (Tabela 5). Analisando os mesmos parâmetros em relação ao pós-operatório, também se observou correlação positiva estatisticamente significante entre os escores da escala "satisfação com correção" e o escore global. Ao se correlacionar estes escores com medidas clínicas (EE e AV s/c no melhor e no pior olho), verificou-se apenas correlação negativa fraca, estatisticamente significante, entre os escores citados e a AV s/c (logMAR) no pior olho, ou seja, quanto menor a $\mathrm{AV} \mathrm{s} / \mathrm{c}$ em $\log \mathrm{MAR}$ no pior olho (melhor visão), mais altos os escores global e de "satisfação" pós-operatórios (Tabela 6).

Tais observações ressaltam que a satisfação com correção, tanto no pré como no pós-operatório, está mais fortemente relacionada aos escores globais do questionário NEI-RQL do que às medidas clínicas rotineiramente utilizadas (EE e AV do melhor e do pior olho). Resultados semelhantes foram relatados por Vitale et al., que verificaram maior correlação entre a melhora no escore de satisfação e a melhora no escore global do RSVP do que entre o ganho em satisfação e as mudanças na AV com e sem correção ou no valor absoluto do erro refrativo ${ }^{(7)}$. Outros autores, em estudo semelhante, corroboram os achados acima, tendo verificado correlação entre questionários de satisfação, qualidade de vida e qualidade de visão, mas não entre estes e medidas de acuidade visual ${ }^{(31)}$.

Estudo independente verificou que o NEI-RQL apresenta maior consistência interna e reprodutibilidade quando comparado ao RSVP, o que nos encoraja a recomendar seu uso para a avaliação da qualidade de vida relacionada à visão em pacientes portadores de erro refrativo, antes e após a realização de cirurgia refrativa ${ }^{(35)}$.

Os autores do questionário NEI-RQL original, diante da sensibilidade do mesmo para detectar mudanças clínicas e na qualidade de vida, decorrentes da cirurgia refrativa, sugerem que sua utilização em estudos futuros possa avaliar o impacto de outras intervenções, como o implante de lentes intra-oculares multifocais ou fácicas, na qualidade de vida dos pacientes ${ }^{(11)}$. Da mesma forma, esperamos que o NEI-RQL possa ser útil, também, na avaliação de novas tecnologias na área de cirurgia refrativa, como a aberrometria e a cirurgia personalizada, analisando os resultados sob o ponto de vista dos pacientes, para que se possa determinar o ganho real em qualidade de vida e de visão proporcionado por estes avanços.

\section{CONCLUSÕES}

Nossa versão em língua portuguesa do questionário NEIRQL mostrou-se bastante sensível para detectar alterações clinicamente significativas decorrentes da cirurgia refrativa. Observou-se melhora da qualidade de vida, refletida no aumento significante dos escores de todas as escalas do questionário, após cirurgia.

\section{ABSTRACT}

Purpose: To assess the impact of refractive surgery on quality of life using the National Eye Institute Refractive Error Quality of Life instrument (NEI-RQL) and to evaluate the responsiveness of our Portuguese version of the NEI-RQL instrument to surgical correction of refractive error. Methods: In this prospective study, the NEI-RQL, a 42-item questionnaire with 13 scales, was self-administered by 96 patients of the Federal University of São Paulo - UNIFESP, between March 2002 and April 2003, before and after undergoing surgical correction of refractive error. An overall scale score was determined by calculating the mean of the 13 scale scores. Differences between preoperative and postoperative NEI-RQL scores were examined. Responsiveness was assessed by calculating the size of effect for each scale of the instrument. Results: Refractive surgery was associated with statistically significant improvements in scores for all the scales of the NEI-RQL. The NEI-RQL has shown to be responsive to surgical correction of refractive error, and the calculated effect sizes ranged from 0.4 ("glare") to 4.19 ("expectations"). Conclusions: The NEI-RQL is highly responsive to changes in vision-related quality of life resulting from keratorefractive surgery. We observed improvements in all the scales of the NEI-RQL, after surgery.

Keywords: Refractive errors/surgery; Laser surgery; Refraction, ocular; Visual acuity; Quality of life; Activities of daily living; Patient satisfaction; Questionnaires

\section{REFERÊNCIAS}

1. Legro MW. Quality of life and cataracts: a review of patient-centered studies of cataract surgery outcomes. Ophthalmic Surg. 1991;22(8):431-43.

2. Kahle G, Seiler T. Wollensak J. Report on psychosocial findings and satisfaction among patients one year after excimer laser photorefractive keratectomy. Refract Corneal Surg. 1992;8(4):286-9.

3. Gimbel HV, Van Westenbrugge JA, Johnson WH, Willerscheidt AB, Sun R, Ferensowicz M. Visual, refractive, and patient satisfaction results following bilateral photorefractive keratectomy for myopia. Refract Corneal Surg. 1993;9 (2 Suppl 1):S5-10.

4. Freitas C, Oliveiros BM, Marques E, Leite EB. Effect of photorefractive keratectomy on visual functioning and quality of life. J Refract Surg. 1995;11 (3 Suppl):S327-S34.

5. McGhee CN, Orr D, Kidd B, Stark C, Bryce IG, Anastas CN. Psychological aspects of excimer laser surgery for myopia: reasons for seeking treatment and patient satisfaction. Br J Ophthalmol. 1996;80(10):874-9.

6. Al-Kaff AS. Patient satisfaction after photorefractive keratectomy. J Refract Surg. 1997;13(5 Suppl):S459-60.

7. Vitale S, Schein OD, Meinert CL, Steinberg EP. The refractive status and vision profile. a questionnaire to measure vision-related quality of life in persons with refractive error. Ophthalmology. 2000;107(8):1529-39.

8. Brunette I, Gresset J, Boivin JF, Boisjoly H, Makni H. Functional outcome and satisfaction after photorefractive keratectomy. Part 1: Development and validation of a survey questionnaire. Ophthalmology 2000;107(9):1783-9.

9. Schein OD, Vitale S, Cassard SD, Steinberg EP. Patient outcomes of refractive surgery. The refractive status and vision profile. J Cataract Refract Surg. 2001;27(5):665-73

10. Berry S, Mangione CM, Lindblad AS, McDonnell PJ. Development of the National Eye Institute Refractive Error Correction Quality of Life Questionnaire. Focus Groups. Ophthalmology. 2003;110(12):2285-91.

11. McDonnell PJ, Mangione C, Lee P, Lindblad AS, Spritzer KL, Berry S, et al. Responsiveness of the National Eye Institute Refractive Error Quality of 
796 Avaliação do impacto da cirurgia refrativa na qualidade de vida por meio do questionário NEI-RQL (National Eye Institute Refractive Error Quality of Life)

Life instrument to surgical correction of refractive error. Ophthalmology 2003;110(12):2302-9.

12. Mangione CM, Phillips RS, Seddon JM, Lawrence MG, Cook EF, Dailey R, et al. Development of the 'Activities of Daily Vision Scale'. A measure of visual functional status. Med Care. 1992;30(12):1111-26.

13. Steinberg EP, Tielsch JM, Schein OD, Javitt JC, Sharkey P, Cassard SD, et al. The VF-14. An index of functional impairment in patients with cataract. Arch Ophthalmol. 1994;112(5):630-8.

14. Massof RW, Rubin GS. Visual function assessment questionnaires. Sury Ophthalmol. 2001;45(6):531-48.

15. Hays RD, Spritzer KL. National Eye Institute Refractive Error Quality of Life Instrument (NEI-RQL-42), Version 1.0: a manual for scoring and use. Los Angeles, Calif: Rand Health Sciences; 2002.

16. Hays RD, Mangione CM, Ellwein L, Lindblad AS, Spritzer KL, McDonnel PJ. Psychometric properties of the National Eye Institute-Refractive Error Quality of Life instrument. Ophthalmology. 2003;110(12):2292-301.

17. Nunes LM, Cotizo V, Schor P. Validação de versão em língua portuguesa do questionário NEI-RQL como instrumento de avaliação da qualidade de vida relacionada à visão, em candidatos a cirurgia refrativa. Rev Bras Oftalmol. 2004;63(2):110-8.

18. Cohen J. Statistical power analysis for the behavioral sciences. 2nd ed. Hillsdale, NJ: Lawrence Erlbaum Associates; 1988.

19. Ferraz EVAP, Lima CA, Cella W, Arieta CEL. Adaptação de questionário de avaliação da qualidade de vida para aplicação em portadores de catarata. Arq Bras Oftalmol. 2002; 65(3):293-8.

20. Atique D, Goulart DG, Lake JC, Lima FA, Felberg S, Nishiwaki-Dantas MC. Qualidade de vida após transplante penetrante de córnea. Arq Bras Oftalmol. 2002;65(3):351-4.

21. Nogueira R, Franca M, Lobato MG, Belfort R, Souza CB, Gomes JAP. Qualidade de vida dos pacientes portadores de síndrome de Stevens-Johnson. Arq Bras Oftalmol. 2003;66(1):67-70.

22. Halliday BL. Refractive and visual results and patient satisfaction after excimer laser photorefractive keratectomy for myopia. Br J Ophthalmol. $1995 ; 79(10): 881-7$
23. Hamberg-Nystrom H, Tengroth B, Fagerholm P, Epstein D, Van der Kwast EM. Patient satisfaction following photorefractive keratectomy for myopia. Refract Surg. 1995;11(3 Suppl):S335-6.

24. Rushood AA, Nassim HM, Azeemuddin T. Patient satisfaction after photorefractive keratectomy for low myopia using the visual analogue scale. J Refract Surg. 1997;13(5 Suppl):S438-40.

25. Shah S, Perera S, Chatterjee A. Satisfaction after photorefractive keratectomy. J Refract Surg. 1998;14(2 Suppl):S226-7.

26. Knorz MC, Wiesinger B, Liermann A, Seiberth V, Liesenhoff H. Laser in situ keratomileusis for moderate and high myopia and myopic astigmatism. Ophthalmology. 1998;105(5):932-40.

27. Houtman DM. Managing patient expectations. Int Ophthalmol Clin. 2000;40(3):29-34.

28. Ben-Sira A, Loewenstein A, Lipshitz I, Levanon D, Lazar M. Patient satisfaction after 5.0-mm photorefractive keratectomy for myopia. J Refract Surg. 1997;13(2):129-34.

29. McGhee CN, Craig JP, Sachdev N, Weed KH, Brown AD. Functional, psychological, and satisfaction outcomes of laser in situ keratomileusis for high myopia. J Cataract Refract Surg. 2000;26(4):497-509.

30. Brunette I, Gresset J, Boivin JF, Pop M, Thompson P, Lafond GP, et al. Functional outcome and satisfaction after photorefractive keratectomy Part 2: survey of 690 patients. Ophthalmology. 2000;107(9):1790-6.

31. Lesueur L, Munoz-Sastre MT, Mullet E, Dabadie B, Arné JL. [Predictors of quality of life in refractive surgery]. J Fr Ophtalmol. 2003;26(7):699-709. French.

32. Kazis LE, Anderson JJ, Meenan RF. Effect sizes for interpreting changes in health status. Med Care. 1989;27(3 Suppl):S178-89.

33. Schein OD. The measurement of patient-reported outcomes of refractive surgery: the refractive status and vision profile. Trans Am Ophthalmol Soc. 2000 98:439-69.

34. Gill TM, Feinstein AR. A critical appraisal of the quality of quality-of-life measurements. JAMA. 1994;272(8):619-26.

35. Nichols JJ, Mitchell GL, Saracino MBA, Zadnik K. Reliability and validity of refractive error-specific quality-of-life instruments. Arch Ophthalmol. 2003;121(9):1289-96. 